\title{
Mechanism, Reduction, and Emergence in Two Stories of the Human Epistemic Enterprise
}

\author{
Paul Teller
}

Received: 9 June 2010/ Accepted: 9 June 2010/Published online: 2 September 2010

(C) The Author(s) 2010. This article is published with open access at Springerlink.com

\begin{abstract}
The traditional way of thinking about science goes back to the corpuscular philosophy with its micro-reductive mechanism and metaphor of reading God's Book of Nature. This "story-1" with its rhetoric of exact truths contrasts with "story-2" which describes science as a continuation of the always imperfect powers of representation given to us by evolution. On story- 2 reduction is one among other knowledge fashioning strategies and shares the imperfections of all human knowledge. When we appreciate that human knowledge always admits of refinement, what appear as "emergent properties" no long seems mysterious.
\end{abstract}

\section{Introduction}

I am going to tell two stories about truth and scientific knowledge. The first will be "historical", the second "biological", where the shudder quotes flag these stories as highly simplified and stylized sketches in which, however, readers will recognize systematic themes from what we know of the messy real world. The first story will include a way of thinking about reduction in terms of mechanism, what has traditionally been called "micro-reduction". Comparing the first and second story will yield a way of thinking about the role of reduction in the larger epistemic enterprise as still important but as one strategy among many, and often functioning as a loose inspirational approach. On the second story human knowledge is always something that stands to be improved, not just by extending it to new subjects but by

\footnotetext{
Many thanks to the Tilberg Conference on Reduction and the Special Sciences, organized by Mark Colyvan, Stephan Hartmann, Maurice Schouten, and Katie Steele, for the opportunity to present and further develop this material; and also to Andrew Wayne for many materially useful comments. Two anonymous referees provided superb comments that prompted a great deal of improvement in the paper.
}

P. Teller $(\bowtie)$

University of California, Davis, CA, USA

e-mail: prteller@ucdavis.edu 
refining it where it already applies. These considerations will then suggest that, when thinking in terms of the second story, phenomena that appear emergent do so naturally and unproblematically, that they are just what one would expect.

\section{Story-1}

Western intellectual culture has long had an obsession with truth, understood as an evaluative characterization of statements as achieving a certain kind of intellectual perfection. A truth involves no error-that presumably goes without saying. But in addition a truth in the sense that $I$ have in mind is thought of as not being refinable. It just states what is, period. One truth can always be supplemented with othersaugmented with representation of further facts. So being all encompassing, not being susceptible to extension with further truths, is not the kind of refinement I have in mind. But truths involving blatantly vague statements can be refined with more precise statements, for example moving from the vague "John is tall" to the precise "John's height is 6 feet precisely". What I take to be a traditional conception of truth, the truths that most of us take ourselves really to be after, do not suffer the defect of being refinable in this kind of way-what I will call "non-refinable" truth. ${ }^{1}$

Like the requirement that truth involve no error, I suspect that the requirement of truth as being non-refinable went without saying. I don't know of any explicit historical discussion of this issue, but I take it to be clearly presupposed in practice, historically speaking. Possibly this way of thinking about human truth and knowledge goes back (at least) to Plato, at least insofar as knowledge of the forms was concerned. Truth thus conceived played an important role in, and must have been encouraged by, the Christian tradition with its omnipotent, omniscient deity.

I want to pick up the story in the Early Modern period with the advent of the so-called "mechanical" or "corpuscular" philosophy. Natural philosophy was to provide truths, in the uncompromising traditional non-refinable sense, of all natural phenomena as described in God's "Book of Nature". More specifically, according to the mechanical philosophy all natural phenomena could be comprehended on the model of understanding the behavior of a mechanical clock. All natural phenomena were to be subsumed in terms of shaped matter in motion and understood by breaking mechanical motions down to the behavior of the smallest material parts. Such accounts needed two kinds of components. On the one hand they worked in terms of analysis of the whole in terms of component material parts. On the other

\footnotetext{
${ }^{1}$ I qualified the claim that truth involves no error as presumably going without saying because, while I agree strictly speaking, I also maintain that the claim is misleading. Often things that we accept as true aren't strictly speaking true but are "true enough" for purposes at hand. Moreover, I take there to be a kind of tradeoff between being precise but merely true enough and being strictly speaking true but not precise-that these function as kind of "semantic alter-egos", so that broadly, evaluation as true really functions as evaluation as being true enough. I have written about these claims elsewhere $(2008$, in preparation). But they are contentious, and for present purposes it will suffice to work with the traditional idea of a kind of "gold standard", unrefinable truth, that not only involves no outright error but in no way stands to be refined.
} 
hand, insofar it was regular or reliable behaviors in which one was interested, one required regularities, rules, or, as they came to be called, natural laws governing the behavior of the parts and their interactions. Together these two components comprise the conception of what we now call "micro-reduction" that requires analysis into parts, the behavior of which is described with exceptionless, universally applicable natural laws, yielding the regularities that we discover in the wholes of our experience. Supplanting Aristotelian analysis of powers, analysis by breaking a whole and its behavior down into the mechanical behavior of its working parts became the standard of explanation. ${ }^{2}$

Reduction will also have a prominent role to play in story-2. Its novel feature in story-1 is its interpretation in terms of non-refinable truth. Speaking in terms of the metaphor of God's Book of Nature, and being God's beyond any possible further refinement, in this book each material event has its exact place in the complete system of events and natural laws, each with determinate causal ties to other events as described by the laws. Since the material events covered all natural phenomena this compendium would be complete-remember that nothing about the future or the past would be hidden from the eyes of Laplace's superintelligence who calculated using only Newton's laws and data about the present. According to what I will call "the doctrine of micro-reduction", each event has its place in this mechanical network so that all natural facts are subsumed by the science of moving parts.

It will be useful to break the doctrine of micro-reduction down into three distinguishable theses:

(a) Scientific accounts proceed by showing how the properties and behavior of things result from the properties and behavior of the parts of things ("analysis in terms of moving parts").

(b) A full account carries the process described by a) down to the level of smallest "ultimate" parts.

(c) It is a humanly achievable objective to provide such accounts with complete precision and accuracy.

I will refer to an account as described by (a) as reduction, as described by (a) and (b) as "micro-reduction", where (c) is then an interpretation placed on micro-reduction.

Over the succeeding three centuries the sciences liberalized this narrowly conceived mechanistic program in one or another respect. In the life sciences one may work with biological rather than material parts, in the social sciences with social or personal or psychological parts, and so on. But many persist in seeing such as stop gap measures, ones that should be eliminable "at least in principle". The whole idea of a part has been stretched in various ways. In the physical sciences one works with parts that prove productive for the topic at hand. ${ }^{3}$ Possibly one can see analysis of a vector into vector components, a wave into Fourier components, as

\footnotetext{
${ }^{2}$ For a general introduction to the "mechanical" or corpuscular" philosophy see Shapin (1996, pp. 30-53 and passim).

3 Auyang (1998) argues that we start with our present understanding of a whole and questions that we have about it that then guide us in dividing the whole up into parts suitable for the problem at hand-what she calls "synthetic microanalysis". See Chapter 6 and throughout much of the book.
} 
parts of a very different, non-material kind, "analytic parts". Liberalizing part/ whole analysis further, broadly one usefully analyzes complex capacities in terms of simpler capacities of components, as exemplified by a computer program or a electronic wiring diagram. ${ }^{4}$ But even in the case of such "functional analysis" many want, "at least in principle", to think of understanding the behavior of the smallest parts in the spirit of some kind of "pushyou pullme" mechanical analysis understood or interpreted in the spirit of story-1. The functional analysis would then apply to give a mechanical account of the whole.

I will return below to the delicate question of whether, or to what extent, such liberalizations should be counted as really abiding by the spirit of story- 1 or whether these innovations count as the development of substantially new methods.

Shortly I will be critical of the micro-reductive picture, thesis (a) and (b) above, in its story-1 interpretation, thesis (c). At the same time I want to emphasize the obvious, that in many ways some kind of reduction has proved to be a fabulously fruitful research strategy. But not of the sort, and not in the way envisioned by story1 ; and it has had to share the stage with many other methods. All these issues will come up in the next two sections.

\section{Story $-2^{5}$}

Story-2 is one of humankind as limited organisms that have evolved in and learned to cope with a complex world. Let's illustrate with our perceptual powers and the kind of knowledge with which perception provides us. Take color vision, for example. We think of our powers of sight as providing us with objective information about determinate intrinsic color properties of external objects-being red, blue, green.... It has turned out that such color objectivism provides a very useful but extremely simplified and inaccurate model for human color vision. Rather than anything like a flat response to a range of reflectancies, our system of color vision works by putting together responses as a function of the outputs of three kinds of color receptors each of which has a frequency response peaked around its dedicated wave length. The color vision of some birds works in terms of four such kinds of receptors so that their "colors" must look incommensurably different from ours. Color, it turns out involves a complex relation involving our perceptual apparatus, the surface reflectancies of objects, and complex aspects of context. ${ }^{6}$

Color vision is just the aspect about complexities of visual perception about which we know quite a bit. Very broadly, perception does not work anything like

\footnotetext{
4 This is Cummins notion of "functional analysis" See Cummins (1975).

5 The later part of story- 2 is just the now oft-told story presented by the modeling literature launched by Nancy Cartwright and Ronald Giere, as an account of the representational output of science. While I have not found explicit citations, I know that the first part of story-2, the connection with our biological background, is familiar to many modelers. What I hope to add to these well-known ideas is the close juxtaposition with story-1; the incorporation of the useful part of story-1-reduction-as an inspirational guide and only one of the many methods of story-2; and, the repercussions of the juxtaposition for thinking about emergence as discussed in Sect. 5 .

${ }^{6}$ See Giere (2006, Chap. 2) for a very useful sketch.
} 
simply transmitting a faithful photograph-like image of a scene before us. This complexity goes far beyond the simple idea of taking an image apart into salient segments (edge detectors, and the like) and then reassembling them into a faithful image. Instead there is an intimate integration with behavioral output, information processing, and other functions. ${ }^{7}$

If in perception nature has cobbled together a complex heuristic for collecting information, the same must be expected about how the mind/brain processes this information. Today cognitive science has worked out only occasional fragments of how this all works, and I won't hide my ignorance of just about all of such details that are known. Nonetheless I think I can safely assume that our larger information processing capacities will suffer limitations analogous to those we know about in perception. Not only do we not have precise "premises" from which to start, what our minds do with these "premises" will be anything but precise deduction or calculation to conclusions logically implied by the "premises", even where thought might be be reconstructable as any sort of inference. Rather, we process information taking shortcuts, making simplifications, interleaving with perception, using all kinds of evolutionarily designed heuristics that give us beliefs that are good enough to get by, not precise conclusions.

It would be miraculous if our various cognitive heuristics all pulled together in forming one unified, consistent body of information. Thus, quite likely our beliefs, our knowledge-that, gets fashioned with highly imperfect methods that involve various kinds of regimentation for different kinds of subject matter, so that as a result we can expect far less than perfect consistency across our larger belief system.

Humans have a great deal of pre-scientific knowledge about the world. But on story-2 this knowledge is badly misdesribed by the metaphor of precise deciphering of fragments of God's error free and perfectly precise Book of Nature. Presumably, shortcomings of perceptual knowledge was something that the authors of the scientific revolution thought they could overcome-witness the concern with illusion-and likewise they thought that they could perfect theoretical knowledge. Modern science has vastly augmented what we know about the world. But by the standard of completely precise deciphering of passages of God's Book of Nature, the project has failed completely. Qualitatively speaking, modern science suffers all the shortcomings of pre-scientific human knowledge.

Modern science has produced a deluge of interesting information, but all of it suffers some defect with respect to the accuracy and/or precision demanded by story-1. Our best fundamental theories in physics are highly idealized, stylized accounts. In many respects they succeed in getting us things we want to know, but, qualitatively speaking, they succeed no more than perception if the standard is a perfectly exact rendering. ${ }^{8}$ Much of physics wears it idealizations on its sleeve. One thinks that more broadly science produces much knowledge whose truth is not qualifiable in any way. Let me illustrate with just one example. In what way might

\footnotetext{
7 To get a taste of just how mind-boggling complex the whole process is, and how distant from anything like faithful image reconstruction, see Churchland et al. (1994) Two papers that survey these issues in contrast with more traditional ways of thinking about perception are Noë (2001) and Hurely (2001).

8 See Teller (2004, pp. 435-438) for some details.
} 
one qualify the truth of the statement that water is $\mathrm{H}_{2} \mathrm{O}$ ? How is this statement anything short of just saying what is, with no possible qualification? Accuracy is granted. But hardly perfect precision. At what temperatures and pressures is water $\mathrm{H}_{2} \mathrm{O}$ ? How many $\mathrm{H}_{2} \mathrm{O}$ molecules are needed for it to count as some water? Water is never pure $\mathrm{H}_{2} \mathrm{O}$, and insofar as we can approach the ideal of purified $\mathrm{H}_{2} \mathrm{O}$ we get a substance with very different properties than the stuff we ordinarily refer to as water. "Water is $\mathrm{H}_{2} \mathrm{O}$ " is not a truth beyond further refinement.

I start from the premise that we do have real knowledge, things like grass is green, water is $\mathrm{H}_{2} \mathrm{O}$, and space-time is curved. These representations give us real information about a world that is independent of us, ${ }^{9}$ and it would be an abuse of language not to count them as real knowledge. But it is not the kind of knowledge to which the authors of the scientific revolution aspired, as do most academics today. Just because the intelligentsia has generally got the analysis of knowledge wrong does not mean that, after all, we have no knowledge. Rather the sensible conclusion is that we have to rethink how to think about knowledge. Knowledge-that is always partial, not just in the sense of being extendible to new subjects, but in being refinable; nonetheless as such it counts as real knowledge of the world. ${ }^{10}$

Let me explain how this works with the map-analogy. I want to know how to get from A to B and to know what the distance is. I get knowledge of the answers to such questions from a reliable map. Note: I did not say (perfect precise) true map, I said reliable map. Indeed there is an out of date use of "true" that just means "reliable". That is, we think of this use as out of date and think of ourselves as using 'true' in a stricter sense, but the standards that we apply for the use of "true" better fit the older usage. Reliable (true) maps provide us with real knowledge. And it is only in this robust sense of knowledge, the only sense that has real application, that we have either prescientific or scientific knowledge of the world. ${ }^{11}$

How on story- 2 should we think about properties? According to story-1, there are real, determinate properties and quantities out there in the world to which we successfully attach predicative terms. While modern philosophy continued the medieval debate between nominalism and property realism, physics went ahead with terms such as 'velocity', 'mass'... and more recently 'atom', 'charge'... as terms that, according to story-1, function in the statement of non-refinable truths, presumably by attachment to determinate natural kinds and properties "out there in the world." Indeed, if such attachments could be secured, the introduced terms would function in the statement of non-refinable truths. Story-2 does not deny that the world may be structured with natural kinds and other sorts of determinate properties-God knows what the world is really like, in all its glorious detail. But

\footnotetext{
9 I mean this expression as neutral about the issues discussed in the realism- antirealism debate. For reasons that will be mentioned in footnote 13, the sketch I am giving of story-2 could be further filled out with different approaches to understanding the nature and function of representation. Throughout I will freely use our usual realist-sounding language — talk about properties, things in the world, and the likebut I do this without prejudice as to whether this should be construed as some kind "hard-headed" realism, some sort of anti-realism, or in the spirit of Arthur Fine's NOA (1987, Chap. 7) .

${ }^{10}$ See Teller (to appear b).

11 Likely exceptions in finite mathematics aside.
} 
story-2 denies that we succeed in such attachment of precise predicative terms. ${ }^{12}$ I have already outlined the case for such failure of exact attachment for the terms that we apply in perceptual knowledge, and thinking through examples supports the same conclusion for predicative terms in science. What is the quantity to which "mass" is supposed to be attached? Not the observer independent quantity of Newtonian physics. In relativity mass is merged with energy, and gravitational mass-energy is not, as seen through the eyes of general relativity, a quantity that has a determinate value in determinate space-time volumes. In quantum field theory mass is a renormalization parameter. The "dressed mass", a creature of our approximative techniques, varies with an "impact parameter", that is how massive a particle looks is a function of how hard you hit it. The "bare mass" is really not part of any theory we now have, as our current quantum field theory's attempts to describe it have it come out as an infinite quantity.

It is more plausible to give a story-2 account of the functioning of "mass" and all our predicative terms. On this story we treat predicative terms as precise components of the models that we construct and use as our ultimate representations of the world. Each such model, as a whole, fits the world as does a map, with less that complete accuracy. What, in such a circumstance, can we say about what in the world corresponds to predicative terms used in such a model? There is nothing in the world which corresponds with complete precision and accuracy to a predicative term, but the world is something like what it would be if it were put together with a property or quantity with just the aspects of its look-alike in the model. ${ }^{13}$

\section{Story-1 as a Source of Methodological Inspiration in Story-2}

Stories-1 and - 2 tell us very different things about what human knowledge is and how the knowledge building enterprise works. Story-1 told of the exact deciphering of at least fragments of the Book of Nature, using the mechanistically inspired methodology of micro-reduction. Story- 2 instead writes various plots and evaluates them on the basis of their explanatory, predictive, and other representational values. There is no need when writing these plots to restrict oneself to just one plot-writing method or style. Any approach that produces good plots gets a place of pride. As such the reductive strategy, precept (a) of story-1, plays a prominent inspirational role.

By pursuing reduction we have produced wonderful accounts of aspects of the world. But the "reduction" of scientific success does not look anything like the

\footnotetext{
${ }^{12}$ This is the reason that my sketch of story-2 is neutral about any issues about realism.

${ }^{13}$ Of course, it can't be ruled out that sometimes we get lucky and there is some kind of exact correspondence between a predicative term in a model and the way the world is actually put together, correspondence at least in the sense that with respect to the term in question there is no further room for correction or refinement. But in a great many cases, perhaps in most, we already know that there is room for just such improvement.

Also note that often a model will grossly distort one feature to facilitate less laborious and more accurate access to others. The use of the point particle idealization in mechanics provides a familiar example. Part of the art of using such models is knowing where and how they apply well, while understanding which aspects constitute radical distortions.
} 
early modern clock metaphor or contemporary philosophers' derivations using bridge laws. At best, accounts of wholes in terms of parts involves severe idealizations at both levels, idealizations about the nature of the relations between wholes and parts, and the understanding in terms of parts usually has to be guided by prior information about the wholes of interest. Efforts to understand continuum hydrodynamics in terms of quantum mechanics illustrates these circumstances. Often what will count as parts is a matter not of metaphysics but of theoretical convenience, regimented by the need for severe idealization at both levels of analysis. Appeal to phonons and consistent field approximations provide examples. $^{14}$ Often treatment in terms of parts leaves any mechanical metaphor completely behind. Treatment of critical phenomena ${ }^{15}$ and the field equations of quantum field theory might be thought to involve parts, in some extremely stretched sense, but in nothing like a mechanical analysis.

Recall that story-1's micro-analysis had two components: Analysis in terms of (ultimate) parts, and treatment of those parts in terms of strict regularities. Over the last 300-400 years the later component has taken on a life of its own in mathematical physics. The extremum techniques of Hamiltonian and Lagrangian mechanics can be applied to systems analyzed in terms of (idealized) parts, but also to wholes with no analysis into parts. The same goes for symmetry methods of group theory and other methods of mathematical physics, for example in general relativity. ${ }^{16}$

Story-2 also embraces holistic methodologies in the study of complex systems in physics, biology, and other subjects. Bechtel's contribution to this volume illustrates how analysis into parts often has to be complemented with large-scale integration effected by complex relations and feedback, not only among the parts but with the environment. The arguments about methodological individualism in sociology are precisely arguments about whether methods in sociology have to be limited to working in the spirit of story-1, and methods stemming from Dirkheim show that there are rich alternatives.

Finally, given the complete failures of most strict reductions, the functional analysis mentioned in Sect. 1 might be most naturally placed in the Aristotelian tradition that corpuscularism was meant to supplant.

Many appear to think that the perceived successes of reduction support the conclusion that reduction is the only, or some kind of ultimate methodology, one that has to apply everywhere, "at least in principle." Any such impression evaporates once one appreciates that analysis in terms of parts has functioned in story-2 not as it was envisioned in story- 1 but as a loose inspiration for a wide range of methods, complemented by many other methods that have nothing to do with parts. More central to my agenda is the point that from the successes of reduction in the foregoing extremely loose sense it does not follow, as many seem to conclude, that we have reason to embrace story-1's precepts (b) and (c) that function as an

\footnotetext{
14 Again, see Auyang (1998) Chapter 6 and passim.

15 See Auyang (1998, pp. 183-184).

16 See my (to appear a) for a sketch of the historical interplay between these two components of mechanism.
} 
interpretation of reduction. The story-1 interpretation does not follow and much of what I have sketched of story- 2 supports the view that in many respects story- 1 does not provide nearly as good a model of reduction as does story- 2 .

Looking for a moment at how story-1 has played out historically also suggests that reduction plays as a loose approach to writing story-2 plots not burdened with the interpretation of precepts (b) and (c). The conviction, broadly by the early 20th century, of the atomic composition of matter looked to fit in well will the ultimate moving parts mechanism of story-1's micro-reduction. But now that we know, first about protons, neutrons and electrons, and then about quarks and gluons, an account that we know is also less than a completely accurate model, one can start to wonder whether there really is any bottom level. More importantly, these "moving parts" look less and less like the corpuscles of the early modern period. They are subject to quantum mechanical uncertainty, superposition, and non-locality. Many interpret them as somehow not being identity-bearing objects, and some prominent field theorists insist that, really, there are no particles, only fields. A field theoretic description involves attribution of properties to space-time points, which leaves the space-time points as the only real candidates for ultimate parts. Relationalists about space-time maintain that there are no space-time points....

I'm not going to attempt to sort out this mess here. ${ }^{17}$ The point is that it is a mess rather than the wild success for story-1 micro-reduction that many imagine has occurred. Reduction has been wildly successful, but only when its results are stripped of the interpretation of precepts (b) and (c), read as part of the script of story-2 and as a very loose guideline suggesting that one try to think in terms of parts of some kind or other in some way or other.

Adherents of story-1 protest at this point that, whatever our limitations, it all boils down to interaction of micro-parts at least "in principle", understood as interpreted in story-1. Given the history of story-1s version of micro-reduction, as summarized just above, this claim is at best unclear and controversial. Even if, for the sake of argument, we should grant it, it has no bearing on the concrete knowledge we have of the world at the present time or anytime soon. ${ }^{18}$

\section{The Puzzle of Emergence}

I contend that the puzzle of emergence (emergent properties, laws) looks very different from within stories- 1 and -2 , that there is really a puzzle only from the point of view of story-1. On many ways of characterizing the idea of emergence, in story- 2 we do talk about the sort of properties and laws that get called emergent, but they are all story- 2 common places. They only look puzzling when thinking in terms of story-1.

\footnotetext{
17 In (1999) I argue that the problems with reduction to a field-theoretic account and the issue of relationalism can be, and can only be sorted out, in present terminology, from the point of view of story- 2 .

18 My verdict on story-1 in the context of story-2 corresponds well with Cartwright's rejection of "fundamentalism", and there is much in common in our reasons. See her (1983) and more recently her (1999) chapter 1, and throughout the book However there is a great deal of difference in detail. In particular, my approach does not either argue for nor appeal to a "dappled world".
} 
Talking in a general way about emergence, let us review what kind of issue seems to be at stake. All the ideas of emergence require a hierarchy of theoretical levels, properties and laws at one level "emerging" from those at a lower level, where the lower level comprises the parts of objects at the higher level. On story-1, the lowest level, the level of ultimate parts, is privileged - the hope of story-1 was that, in the (temporal or logical) end, everything would be characterizable in terms of the level of ultimate parts. Interpreters, such as Mill and Broad, were wrestling with the problem that apparently the chemical properties and laws of chemistry did not seem to microreduce to those of fundamental physics, to say nothing of any hope for micro-reduction of theories on up the part-whole hierarchy. (As with my reconstruction of story-1 itself, I am sketching a very simplified version of a much more complex actually history.) The expectation of story-1 seemed threatened! How then did chemistry and theories at higher levels fit into the "natural order"? These theories and their concepts seemed somehow incommensurable with a theory of the ultimate parts-they "emerged" from the lowest level in some way that, by the expectations laid down in story-1, appeared to transcend any scientific understanding.

All this is going to look very different from the point of view of story-2. On story-2 any theory of ultimate parts has no privileged expectation-generating status. Thus, when the properties and laws of chemistry and of higher level theories stubbornly resist efforts at micro-charcterization, this is just what is to be expected, not something that flies in the face of basic expectations. ( $\mathrm{I}$ am here using 'characterization' as an umbrella term that I will refine in a moment.) Story-2 will see the same or similar inter-theoretic relations that story-1 sees, but these relations won't have the same disturbing aspect.

So the shocking aspect to what we think of as the properties and laws that got labeled as "emergent" is an artifact of the privileged states of story-1's expectations for scientific accounts in terms of ultimate parts.

A more careful formulation will require a little work. ${ }^{19}$ First of all, any relation of emergence will involve properties and laws of different theoretical levels, where the notion of level in question is that of parts to wholes. So if we are to look for emergence-analogs in story-2 this will have to involve relations of properties and laws of parts to those of wholes that the parts compose. I will carry the discussion forward just for parts. Very similar comments will go for laws.

Now to be more specific about different ways of understanding "characterization", and therein different ways of understanding 'emergence'. We can sidestep the problem about properties by working in the formal as opposed to the material mode of description. A concept or predicate at one level emerges "conceptually" from the level of the parts if the predicate or concept in the theory of the wholes cannot be

\footnotetext{
19 I won't discuss two useful notions that should be entirely unproblematic on any story. A nominally emergent property, on various presentations, is a property of a whole that is not, or cannot be, a property of its parts, or the kind of property that cannot be the kind of property that it's parts can have. Watches, and generally functional artifacts will generally be nominally emergent. See Bedau (2008a, p. 158). The idea of weak emergence applies to processes that can be reduced to the behavior of parts, but only by an exhaustive description. On Bedau's rendering, weakly emergent processes have "incompressible" characterizations, that is an exhaustive step by step description or calculation cannot be replaced with a shorter description. See Bedau (2008b, p. 445).
} 
reduced to ones in the theory of the parts. "The theory" and "reduced" are parameters here that can be made more precise in various ways, with correspondingly different ways of understanding conceptual emergence. "The theory" could be taken to be theory we now have, or some "final" or "ultimate" theory, one that describes all the facts in its domain with complete precision and accuracy. If the theory is the one we now have, story-2 leads us to expect that often there will be conceptual emergence as just described. In practice theories of different levels may make different modeling assumptions that will employ different conceptual tools that won't fit together in any natural way. On the other hand, if the theory in question is supposed to be the "ultimate theory" it is in the spirit of story-2 not to engage in the discussion. Story-2 is a story about the science we now have and about how we can sensibly expect it to develop in the "foreseeable" future. That "the ultimate theory", should look anything like what story-1 expects, or even that it makes sense to talk about any such thing, is pure faith. The only reason given for this expectation is the claimed "success" of story-1 to date. But the claims are often not born out when more closely examined and, in any case, are more natural understood in terms of story-2, as I explained in Sect. 4.

On the other hand we can restate all these ideas in the material mode. Then the claims are not about our representation of things but about the things represented. This corresponds to what is called "ontological emergence". As far as I can see, this notion is obscure. Sometimes it is simply characterized as "ontological irreducibility" (e.g., Humphreys 2008, p. 586) or various combinations of some kind of irreducibility, supervenience, failure of supervenience, unanalyzed causal relations or an unanalyzed relation of "fusion" (O'Connor and Wong 2006, Sect. 2). I will let readers satisfy themselves, in considering specific proposals for understanding "ontological" emergence, that any real analysis falls back on the prospect for conceptual connections involved in conceptual emergence. In addition, the view of story-2 that our predicates themselves have imprecise connections with the world makes the situation worse for providing a clear notion of what might be meant for properties at one level somehow to be reduced to properties at a lower level. The looseness of fit between predicates and the world provides another reason for expecting that properties-as-explicitly-represented with our imprecise predicative terms in theories at one level do not need to fit nicely into the also imprecise theories we have about their parts.

Finally, emergence can also be understood as some kind of failure of predictability: Knowing facts and the theory of the parts would not enable one to predict facts described using predicates used only in models of the whole, where again, 'theory' might refer to theory we have now, in which case failure of predictability among inexact models will be a common place, or to some "ultimate" theory in which story-2 again takes the question to be beyond any useful discussion.

As just characterized predictive-emergence sounds very like conceptual emergence. The underlying intuitive idea, however, appears to be that people who are familiar with the facts about the parts would not expect, or would be surprised by the properties of the whole that then count as emergent. Primary among such plausible cases are the secondary qualities and states of consciousness, and mental states generally. Broad took such cases to be decisive (1925, pp. 71-72). Put this 
way the issue appears to be quite independent of whether we are thinking in terms of story-1 or -2 . But such independence is called into question by so-called "mode of access" considerations. Space does not allow a detailed examination here, but briefly these considerations suggest that we are surprised because we know about conscious states "subjectively", understood in terms of the knowing subject being in the state in question, while a scientific account of conscious states would be "objective", that is described from an external point of view. Mode of access arguments contend that the subjective and objective access to mental states are two different modes of access to the same states, though the different modes of access make it appear that different states or properties are in question. If this is right, the "surprise" about mental states shows nothing about their status as objects of scientific study. ${ }^{20}$

Altogether, story-2 will talk of many inter-theoretic relations that are, or are close analogs of, relations described within story-1 as emergent. The difference is that in story-2 they are not in any way in conflict with story-2's expectations. Such intertheoretic relations arise when modeling of facts about wholes cannot be redone, in any of many possible ways, in terms of the modeling of the parts, and this because the modeling at the two levels proceeds with different objectives and standards of accuracy and so with very different idealizations and other modeling assumptions that cannot fit together. Not always of course! Understanding and refining intertheoretic relations is always an excellent scientific project. Seeing to what extent one can understand theoretical consequences at one level by appealing to theory at a lower level is always a good scientific question. Many such intertheoretic studies are extremely valuable. Although hydrodynamics can't in any useful sense be reduced to quantum mechanics, there are considerations from quantum mechanics that can be deployed in helping to understand why the continuum idealizations of hydrodynamics work as well as they do. Many so-called successes of unification fit this sort of quite loose description rather anything that would plausible be called "reduction". ${ }^{21}$

I conclude that those who find the emergence candidates puzzling or philosophically problematic are, in effect, working within story-1, in particular, trying to answer questions about the structure of a final, completely accurate and general theory. Insofar as one finds story-2 as a natural way to understand science such questions loose their interest.

\section{Epilogue}

Both story-1 and story-2 are efforts to describe the human epistemic enterprise. As a modeler I'm not going to say that story-1 has no useful application. For example some will continue to use it as a kind of regulative ideal. But story-1 only applies, at

\footnotetext{
${ }^{20}$ Among many, many presentation of such mode of access arguments see my (1992). For a comprehensive survey and discussion see Spencer (2009).

21 See my (to appear a) The integration of electric and magnetic effects in Maxwell's theory and their reduction in special relativity provide notable exceptions.
} 
best, to a kind of "in principle" account. Insofar as our interest is in understanding and interpreting the science we have today, or any remotely like it with respect to its limitations, story- 2 gives a much better fit in a great many respects. I have here given no more than a bare plot outline of these stories and how they have interacted. But I hope that this outline will encourage a very different way of thinking about science than that presupposed by traditional ideas of mechanism, reduction, and emergence.

Open Access This article is distributed under the terms of the Creative Commons Attribution Noncommercial License which permits any noncommercial use, distribution, and reproduction in any medium, provided the original author(s) and source are credited.

\section{References}

Auyang, S. Y. (1998). Foundations of complex-systems theories in economics, evolutionary biology, and statistical physics. Cambridge: Cambridge University Press.

Bechtel, W. (this issue). The downs and ups of mechanistic research: Circadian rhythm research as an exemplar. (this issue).

Bedau, M. (2008a). Downward causation and autonomy in weak emergence. In M. A. Bedau \& P. Humphreys (Eds.), Emergence: Contemporary readings in philosophy and science. Cambridge: MIT Press.

Bedau, M. (2008b). Is weak emergence just in the mind? Minds \& Machines (2008), 18, 443-459.

Broad, C. D. (1925). Mind and its place in nature. London: Routledge and Kegan Paul.

Cartwright, N. (1983). How the laws of physics lie. Oxford: Oxford University Press.

Cartwright, N. (1999). The dappled world. Cambridge: Cambridge University Press.

Churchland, P., et al. (1994). A critique of pure vision. In C. Koch \& J. L. Davis (Eds.), Large-scale neuronal theories of the brain. Cambridge: Bradford.

Cummins, R. (1975). Functional analysis. Journal of Philosophy, 72, 741-764.

Feigl, H. (1971). "Some crucial issues of mind-body monism" as reprinted in: Feigl, H. (1981). Inquires and provocations, selected writings, 1929-1974. R. S. Cohn (Ed.). Dordrecht: Reidel.

Fine, A. (1987). The shaky game. Chicago: University of Chicago Press.

Giere, R. (2006). Scientific perspectivism. Chicago: University of Chicago Press.

Humphreys, P. (2008). Computational and conceptual emergence. Philosophy of Science, 75, 584-594.

Hurely, S. (2001). Perception and action: Alternative views. Synthese, 129, 2-40.

Noë, A. (2001). Experience and the active mind. Synthese, 129, 41-60.

O'Connor, T., \& Wong, H. Y. (2006). "Emergence" stanford encyclopedia of philosophy. Metaphysics Research Lab, CSLI, Stanford University.

Shapin, S. (1996). The scientific revolution. Chicago: University of Chicago Press.

Spencer, D. I. (2009). Time, tense, and mind: A case study in metaphysics and perspectival representation. Dissertation. University of California at Davis.

Teller, P. (2004). How we dapple the world. Philosophy of Science, 71, 425-447.

Teller, P. (in preparation). Some dirty little secrets about truth.

Teller, P. (to appear a). "Unity and disunity in science" To appear in the new Macmillan Encyclopedia of Philosophy.

Teller, P. (to appear b). Provisional knowledge. 